\title{
Groups with a recursively enumerable irreducible word problem
}

\author{
Gabriela Aslı Rino Nesin and Richard M. Thomas * \\ Department of Computer Science, University of Leicester, Leicester LE1 7RH, U.K.
}

\begin{abstract}
The notion of the word problem is of fundamental importance in group theory. The irreducible word problem is a closely related concept and has been studied in a number of situations; however there appears to be little known in the case where a finitely generated group has a recursively enumerable irreducible word problem. In this paper we show that having a recursively enumerable irreducible word problem with respect to every finite generating set is equivalent to having a recursive word problem. We prove some further results about groups having a recursively enumerable irreducible word problem, amongst other things showing that there are cases where having such an irreducible word problem does depend on the choice of finite generating set.
\end{abstract}

\section{Introduction}

One area where computer science interfaces with important concepts in mathematics is in the consideration of word problems of groups as formal languages. The first questions considered here concerned solvability; the word problem was shown to be undecidable for finitely presented groups by Novikov [16] and Boone [2]; so a finitely presented group can have a word problem that is recursively enumerable but not recursive. Algebraic characterizations have been given for groups with a recursively enumerable word problem in [10] and a recursive word problem in [3].

Attention then turned to considering groups with word problems in simpler classes of languages. A characterization of groups with a regular word problem was given in [1] and then the same was done (assuming a subsequent deep result of Dunwoody [4]) for groups with a context-free word problem in [15]. Stating that the word problem lies in a particular class of languages is often (as in the cases mentioned here) independent of the choice of finite generating set (see Remark 1 below).

In this paper we consider the irreducible word problem of a group; this is the set of words in the word problem $W$ which have no non-empty

\footnotetext{
* The second author would like to thank Hilary Craig for all her help and encouragement.
} 
proper subwords in $W$ (see Definition 7). The irreducible word problem is intrinsically connected with the word problem; using the terminology of [11] we have that $W$ is the insertion closure of the irreducible word problem $I$ together with the empty word $\{\epsilon\}$ whereas $I$ is the insertion base of $W$ (see [6] for further details).

The notion of an irreducible word problem was introduced in [8] where groups with a finite irreducible word problem were studied. The history of the study of the irreducible word problem has, in some sense, followed that of the word problem in the reverse direction; with the word problem (as mentioned above) the initial considerations were with groups with a recursive or recursively enumerable word problem and the cases where the word problem lay in a more restricted class of languages came later. The study of the irreducible word problem started with groups with a finite irreducible word problem in [8], which was continued in $[17,18]$; it was then pointed out in [6] that there are no groups whose irreducible word problem is regular but not finite. There are also some interesting connections with string rewriting systems as explained in [14]. Groups with a context-free irreducible word problem were considered in [5-7] and with a context-sensitive irreducible word problem in [12].

In the case of recursive languages it was shown in [7] that the irreducible word problem of a group is recursive if and only if the word problem is recursive. As we point out in Remark 9, this gives an example where the irreducible word problem lying in a class $\mathcal{F}$ of languages is independent of choice of finite generating set for the group in question. While this also holds for context-sensitive languages (see [12]) it does not hold in general even when $\mathcal{F}$ is closed under inverse homomorphism, which is a particular complication when studying irreducible word problems.

In this paper we take the next natural step by considering groups which have a recursively enumerable irreducible word problem; this is a situation where membership can depend on choice of finite generating set (see Corollary 14). Our main result (Theorem 12) considers the case where we do have independence and is rather surprising: having a recursively enumerable irreducible word problem with respect to every finite generating set is equivalent to having a recursive word problem. In such groups having a recursively enumerable irreducible word problem and having a recursive irreducible word problem are equivalent, which is in complete contrast to the situation for word problems as mentioned above. This equivalence is not the case for all groups, however (Proposition 13).

The structure of the paper is as follows. In Section 2 we give the necessary background material from group theory. In Section 3 we introduce 
the notion of a loopy group (Definition 2) which plays a fundamental role in proving our results. We show that any group is loopy with respect to some finite generating set (Theorem 5) and give an alternative characterisation of loopiness (Theorem 6). In Sections 4 and 5 we consider irreducible word problems building on previous work of the second author with Fonseca in $[5,7]$. As we have mentioned, we establish our main result in Section 4 and show that having a recursively enumerable irreducible word problem can depend on choice of finite generating set in Section 5 .

\section{Background from group theory}

For the convenience of the reader we will summarize here some notions from group theory we will need in this paper and the notation we will be using.

If $X$ is an alphabet then we say that $X$ is a monoid generating set for a group $G$ if we have a surjective homomorphism $\varphi: X^{*} \rightarrow G$; informally every element of $G$ can be written as a word in $X^{*}$. If $A$ is an alphabet and we let $\Sigma=A \cup A^{-1}$, where $A^{-1}$ is the alphabet $\left\{x^{-1}: x \in A\right\}$ in a (1-1) correspondence with $A$, and if we have a surjective homomorphism $\varphi: \Sigma^{*} \rightarrow G$ (where, if $x \in A$, we insist that $x^{-1} \varphi=(x \varphi)^{-1}$ ), then $A$ is said to be a group generating set for $G$. We will only consider group generating sets in this paper (and so we will simply refer to "generating sets" from now on). Note that $\Sigma=A \cup A^{-1}$ is formally a set of characters, not a subset of $G$, although it will sometimes be convenient to associate a word $\alpha \in \Sigma^{*}$ with the element $\alpha \varphi$ of $G$ which it represents (i.e. to suppress the reference to $\varphi$ ).

We have the correspondence $A \rightarrow A^{-1}$ defined by $x \mapsto x^{-1}$. If $x \in A$ we let $\left(x^{-1}\right)^{-1}$ be $x$; this extends our correspondence to a bijective map from $\Sigma$ to $\Sigma$. We extend this to a bijective map from $\Sigma^{*}$ to $\Sigma^{*}$ by defining

$$
\epsilon \mapsto \epsilon \text { and } x_{1} x_{2} \ldots x_{n} \mapsto x_{n}^{-1} \ldots x_{2}^{-1} x_{1}^{-1}
$$

for $x_{i} \in \Sigma$, and so we may write $\alpha^{-1}$ for any $\alpha \in \Sigma^{*}$. If $\alpha \varphi=g$ for $\alpha \in \Sigma^{*}$ then it follows that $\alpha^{-1} \varphi=g^{-1}$.

If $\alpha, \beta \in \Sigma^{*}$ then we will normally write $\alpha=\beta$ if $\alpha$ and $\beta$ are identical as words and $\alpha={ }_{G} \beta$ if $\alpha$ and $\beta$ represent the same element of $G$ (i.e. if $\alpha \varphi=\beta \varphi)$.

A group presentation $\langle A: R\rangle$ for a group $G$ consists of a generating set $A$ for $G$ and a set $R$ of words over $\Sigma=A \cup A^{-1} ; R$ must satisfy the property that the group $G$ is isomorphic to $\Sigma^{*} / \approx$ where $\approx$ is the congruence on $\Sigma^{*}$ generated by all the pairs of the form $(w, \epsilon)$ with $w \in R$, 
together with all the pairs of the form $\left(x x^{-1}, \epsilon\right)$ and $\left(x^{-1} x, \epsilon\right)$, where $x \in A$. The word problem $W_{A}(G)$ for $G$ with respect to $A$ consists of the set of all the words in $\Sigma^{*}$ that represent the identity element $1_{G}$ of $G$.

Remark 1. It is well known (see [9] for example) that, if $\mathcal{F}$ is a family of languages closed under inverse homomorphism, then $W_{A}(G)$ lying in $\mathcal{F}$ is independent of the choice of finite generating set $A$ for a finitely generated group $G$. A particular case is where $\mathcal{F}$ is the family of recursive languages, so that $G$ having a solvable word problem is independent of the choice of finite generating set.

If $G$ is a group generated by a finite set $A$ then we define the Cayley graph $\Gamma=\Gamma(G, A)$ to be the graph whose vertices are the elements of $G$ and where we have, if $x \in \Sigma$, a directed edge labelled by $x$ from $g$ to $h$ if $g x={ }_{G} h$ (we sometimes write $g \stackrel{x}{\rightarrow} h$ here). We will refer to the identity element of $G$ as being the origin of the graph $\Gamma$.

Given that the edges of $\Gamma$ are labelled by elements of $\Sigma$, directed paths in $\Gamma$ are labelled by elements of $\Sigma^{*}$; we talk about the path from $u$ to $v$, or the cycle starting at $u$, as having the label $\alpha$. Here a path from $u_{1}$ to $u_{n}$ is a sequence of vertices $u_{1}, u_{2}, \ldots, u_{n}$ such that $u_{i}$ is adjacent to $u_{i+1}$ for $1 \leqslant i \leqslant n-1$ and a cycle starting at $u_{1}$ is such a path where $u_{1}=u_{n}$; cycles and paths are directed. Such a path is said to be simple if the vertices $u_{1}, u_{2}, \ldots, u_{n}$ are all distinct and a cycle is said to be simple if the vertices $u_{1}, u_{2}, \ldots, u_{n-1}$ are all distinct.

\section{$3 \quad$ Loopy groups}

We now introduce the idea of a "loopy group" which will play a central role in establishing our results. To avoid trivial situations we confine our attention to groups with at least three elements (this is not a real restriction; when talking about groups with a recursively enumerable irreducible word problem we are really only interested in infinite groups anyway).

Definition 2. Let $A$ be a finite generating set for a group $G$ with $|A|>1$ and $|G|>2$ and let $\Sigma=A \cup A^{-1}$. $G$ is said to be loopy with respect to $A$ if, for any $a, b \in \Sigma$ such that $a b^{-1} \neq_{G} 1_{G}$, there is a simple cycle in $\Gamma(G, A)$ starting at $1_{G}$ labelled by $a c_{1} \ldots c_{m} b^{-1}$ for some $c_{1}, \ldots, c_{m} \in \Sigma$.

Remark 3. In other words, if a group is loopy, then there is a way to get from any vertex $a$ at distance one from the origin in $\Gamma(G, A)$ to any other such vertex $b$ without passing through the origin (if there is a path from 
$a$ to $b$ that does not pass though the origin then there is a simple such path $P$ and then the cycle that goes from $1_{G}$ to $a$, then follows $P$, and finishes by going from $b$ to $1_{G}$ is a simple cycle of the required form). The converse of this also holds. We will use this equivalent formulation of loopiness in what follows.

Remark 4. If $G$ is loopy with respect to a finite generating set $A$ then, for any edge in $\Gamma=\Gamma(G, A)$, there is a simple cycle containing it. To see this consider an edge $e$ with label $a \in \Sigma=A \cup A^{-1}$ joining $g$ to $h$. There is then an edge joining $1_{G}$ to $a=g^{-1} h$. If we pick some other element $b$ of $\Sigma$ with $a b^{-1} \neq_{G} 1_{G}$ then, by the definition of loopiness, there is a simple cycle in $\Gamma$ starting at $1_{G}$ labelled by $a c_{1} \ldots c_{m} b^{-1}$ for some $c_{1}, \ldots, c_{m} \in \Sigma$. The word $a c_{1} \ldots c_{m} b^{-1}$ also labels a simple cycle starting at $g$ where the first edge in the cycle is $e$ as required.

A critical fact is that any finitely generated group is loopy with respect to some finite generating set:

Theorem 5. For any finitely generated group $G$ with $|G|>2$ there is a finite generating set $A$ with respect to which $G$ is loopy.

Proof. Let $G=\langle B: R\rangle$ be a presentation of $G$ where $B$ is finite. We extend the generating set $B$ as follows: for any two elements $a, b \in B \cup B^{-1}$ such that $a^{-1} b \notin B \cup B^{-1} \cup\left\{1_{G}\right\}$ we define a new generator $z_{a^{-1} b}$ where $z_{a^{-1} b}={ }_{G} a^{-1} b$. Let

$$
A=B \cup\left\{z_{a^{-1} b}: a, b \in B \cup B^{-1}, a^{-1} b \notin B \cup B^{-1} \cup\left\{1_{G}\right\}\right\}
$$

and

$$
S=R \cup\left\{z_{a^{-1} b}=a^{-1} b\right\} .
$$

It is clear that $\langle A: S\rangle$ is another presentation for $G$; we will show that $G$ is loopy with respect to $A$. As we commented in Remark 3 all we need to show is that, for any two distinct elements in our new set of generators $A$, there is a path from one to the other in $\Gamma=\Gamma(G, A)$ not passing through the origin. We split our consideration up into three cases.

1. If both the generators $a$ and $b$ are in $B$, then $z_{a^{-1} b}$ labels a simple path from $a$ to $b$ and we are done.

2. Next let us assume that one of the generators $b$ is in $B \cup B^{-1}$ and that the other generator is in $A-\left(B \cup B^{-1}\right)$; so the other generator is of the form $z_{a^{-1} c}$ for some $a, c \in B \cup B^{-1}$.

(a) If $b \neq a^{-1}$ then $z_{b^{-1} a^{-1}} c$ labels a path from $b$ to $z_{a^{-1} c}$ which does not pass through the origin and we are done. 
(b) If $b=a^{-1}$ then $c$ labels a path from $b$ to $z_{a^{-1} c}$ and we are done.

3. Finally let us assume that both the generators are in $A-\left(B \cup B^{-1}\right)$; call them $z_{a^{-1} b}$ and $z_{c^{-1} d}$.

(a) If $a \neq c$ then $b^{-1} z_{a c^{-1}} d$ labels a path from $z_{a^{-1} b}$ to $z_{c^{-1} d}$ and we are done.

(b) If $a=c$ then $z_{b^{-1} d}$ labels a path from $z_{a^{-1} b}$ to $z_{c^{-1} d}$ and we are done.

We have covered all the possibilities and so the result is established.

If $G$ is a group with a finite generating set $A$ we define a property $P_{A}$ on $G$ as follows: if $g \in G$ then $P_{A}(g)$ means there is a simple cycle passing through $1_{G}$ and $g$ in $\Gamma(G, A)$. We now have the following characterisation of loopiness:

Theorem 6. Let $G$ be a group with a finite generating set $A$; then $G$ is loopy with respect to $A$ if and only if $P_{A}(g)$ holds for all $g \in G$.

Proof. $(\Rightarrow)$ Assume that $G$ is loopy. If $a \in \Sigma=A \cup A^{-1}$ then, as in Remark 4 , there is a simple cycle containing the edge joining $1_{G}$ to $a$ in $\Gamma=\Gamma(G, A)$ with label $a$; hence $P_{A}(a)$ holds. Given that $\Gamma$ is connected, to prove that $P_{A}(g)$ holds for all $g \in G$ it is sufficient to prove the following:

if $P_{A}(g)$ does not hold and $h$ is a neighbour of $g$ in $\Gamma$ then $P_{A}(h)$ does not hold.

So assume that $P_{A}(g)$ does not hold and that $h$ is a neighbour of $g$ in $\Gamma$. Assume (for a contradiction) that $P_{A}(h)$ does hold and let $L_{1}$ be a simple cycle passing through $1_{G}$ and $h$ in $\Gamma$. Let $a$ be the label of the edge from $h$ to $g$ in $\Gamma$.

Choose a neighbour $k$ of $h$ on $L_{1}$ and let $b$ be the label of the edge from $h$ to $k$. As $P_{A}(g)$ does not hold we have that $g$ does not lie on $L_{1}$ and so $b$ is distinct from $a$. Since $G$ is loopy there is a simple cycle starting at $1_{G}$ with label of the form $a c_{1} c_{2} \ldots c_{n} b^{-1}$. Premultiplying the vertices of the cycle by $h$ gives a simple cycle $L_{2}$ starting at $h$ with the same label; the first vertex (after $h$ ) on the cycle is $h a=g$ and the last vertex (before $h$ is reached again) is $h b=k$.

Let $m$ be the first vertex on $L_{2}$ after $g$ that lies on $L_{1}$ (see Figure 1); such a vertex must exist as $k$ lies on $L_{1}$. Let $P$ be the subpath of $c_{1} \ldots c_{n}$ between $g$ and $m$. By our choice of $m, P$ does not intersect $L_{1}$ at any vertex except $m$. Therefore, to get a simple loop containing $1_{G}$ and $g$, all 


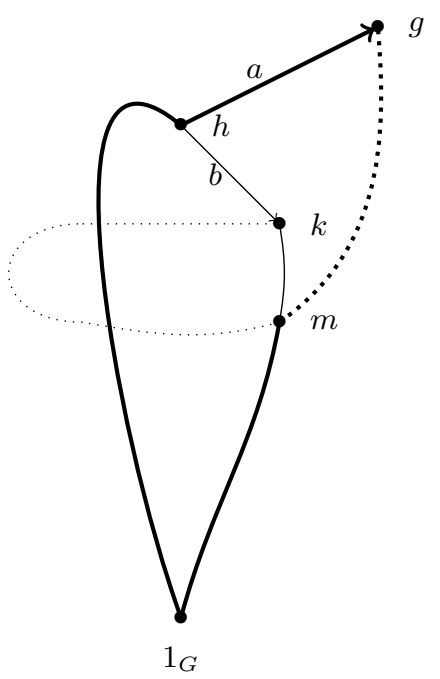

Fig. 1. The path in bold indicates the simple loop through $g$; solid lines represent $L_{1}$ and dotted lines represent $P$.

we need to do is replace the portion of $L_{1}$ between $h$ and $m$ by the edge from $h$ to $g$ followed by $P$. This contradicts the fact that $P_{A}(g)$ does not hold.

$(\Leftarrow) \quad$ Let $a$ and $b$ be any two elements of $\Sigma$ with $a b^{-1} \neq_{G} 1_{G}$. We know that $P_{A}\left(a^{-1} b\right)$ holds and so there are two simple paths $\sigma$ and $\tau$ to the vertex $a^{-1} b$ in $\Gamma$ which only have the vertices $1_{G}$ and $a^{-1} b$ in common. The vertex $a^{-1}$ can only lie on one of these two paths and so we may assume that $\sigma$ does not contain $a^{-1}$. Since $\sigma$ is a path from $1_{G}$ to $a^{-1} b$ not containing $a^{-1}$, premultiplying each vertex on the path by $a$ gives a path (with the same label as $\sigma$ ) from $a$ to $b$ not containing $1_{G}$. As in Remark 3 we see that this ensures that $G$ is loopy.

\section{Irreducible word problems}

We now come to the main topic of this paper:

Definition 7. Let $A$ be a finite generating set for a group $G$ and let $\Sigma=A \cup A^{-1}$. The irreducible word problem $I_{A}(G)$ of $G$ with respect to $A$ is the set of words $w \in W_{A}(G)$ such that

$$
w=\alpha u \beta \text { and } \alpha \beta \neq \epsilon \text { and } u \in W_{A}(G) \Longrightarrow u=\epsilon .
$$


In other words $I_{A}(G)$ is the set of words $w \in W_{A}(G)$ which have no nonempty proper subwords belonging to $W_{A}(G)$. Note that we use the term "subword" of a word $w$ in this paper to denote a sequence of consecutive characters from the word $w$ (as opposed to an arbitrary subsequence of $w$ ).

Just as the word problem for a group $G$ with respect to a finite generating set $A$ can be identified with the set of labels of cycles in the Cayley graph $\Gamma=\Gamma(G, A)$ which start and end at $1_{G}$, the irreducible word problem can be identified with the labels of the simple cycles starting and ending at $1_{G}$.

We need the following result from [7] (see Theorem 4.4 there):

Proposition 8. If $G$ is a group and $A$ is a finite generating set for $G$ then $I_{A}(G)$ is recursive if and only if $W_{A}(G)$ is recursive.

Remark 9. If $\mathcal{F}$ is a family of languages then the fact that the irreducible word problem of a group $G$ with respect to a finite generating set $A$ belongs to $\mathcal{F}$ depends on the choice of $A$ even if $\mathcal{F}$ is closed under inverse homomorphisms, in contrast to the situation with the word problem (see Remark 1 above). However Proposition 8 shows that this is not the case if $\mathcal{F}$ is the class of recursive languages.

We also need the following result from [5] (see Proposition 8.3.1 there):

Proposition 10. Let $G$ be a group and $A$ be a generating set for $G$. If $I_{A}(G)$ is recursively enumerable then $W_{A}(G)$ is recursively enumerable.

Proof. Assume that $I=I_{A}(G)$ is recursively enumerable.

Let $\Sigma=A \cup A^{-1}$ and let $\mathfrak{A}$ be an algorithm that, when given an input $\alpha \in \Sigma^{*}$, terminates if and only if $\alpha \in I$. We outline an algorithm $\mathfrak{A}^{\prime}$ which, given an input $\alpha \in \Sigma^{*}$, terminates if and only if $\alpha \in W=W_{A}(G)$.

We apply $\mathfrak{A}$ to $\alpha$ and every one of its non-empty proper subwords. If $\mathfrak{A}$ does not terminate on any of these words, then $\alpha$ is not in $W$ and $\mathfrak{A}^{\prime}$ does not terminate either. If $\mathfrak{A}$ terminates on $\alpha$ (in which case $\alpha \in I \subset W$ ), then $\mathfrak{A}^{\prime}$ terminates. If $\mathfrak{A}$ terminates on some non-empty proper subword $u$, say $\alpha=\beta u \gamma$, then $u$ is deleted from $\alpha$. The following procedure is then repeated:

(A) The algorithm $\mathfrak{A}$ is applied to every non-empty subword of the leftover word. If $\mathfrak{A}$ terminates on some such non-empty subword $u^{\prime}$, then $u^{\prime}$ is deleted.

The algorithm $\mathfrak{A}^{\prime}$ terminates with the empty word $\epsilon$ if and only if $\alpha \in W$. If, on some iteration of (A), $\mathfrak{A}$ does not terminate on any nonempty subword, then $\mathfrak{A}^{\prime}$ does not terminate either. 
The following is important for establishing our main result:

Theorem 11. Let $G$ be a group generated by a finite set $A$ such that $P_{A}(g)$ holds for all $g \in G$ in the Cayley graph $\Gamma(G, A)$. Then

$$
I_{A}(G) \text { is recursively enumerable } \Rightarrow W_{A}(G) \text { is recursive. }
$$

Proof. Assume that $I=I_{A}(G)$ is recursively enumerable, so that we have an algorithm $\mathfrak{A}$ which terminates if and only if its input is in $I$.

By Proposition 10 we know that $W=W_{A}(G)$ is recursively enumerable and so we have an algorithm $\mathfrak{B}$ which terminates if and only if its input is in $W$. We now want an algorithm $\mathfrak{B}^{\prime}$ which terminates if and only if its input is not in $W$.

Let $\Gamma=\Gamma(G, A)$ and $\Sigma=A \cup A^{-1}$. If $w$ is any word in $\Sigma^{*}-W$ then, since $P_{A}(w)$ holds, we may choose a simple cycle containing $1_{G}$ and $w$ in $\Gamma$. If $v$ is the label of the simple path from $1_{G}$ to $w$ and $u$ the label from $w$ back to $1_{G}$ on that cycle then we see that $w v^{-1} \in W$ and that $v u \in I$.

Our algorithm $\mathfrak{B}^{\prime}$ which terminates if and only if its input $w$ is not in $W$ proceeds as follows:

(A) Start enumerating words which have $w$ as a proper prefix; for each such word $\alpha$ we enumerate, we start $\mathfrak{B}$ on $\alpha$ which will terminate if $\alpha$ is in $W$. This successively generates the non-empty words $v^{-1}$ such that $w v^{-1} \in W$.

(B) For each such word $v^{-1}$ we generate in (A) we start enumerating words $v u$ which have $v$ as a proper prefix; for each such word $v u$ we enumerate, we start $\mathfrak{A}$ which will terminate if $v u \in I$.

As above, if $w \notin W$, then such non-empty words $v$ and $u$ must exist and, once we have generated them and confirmed that $v u \in I$, then we know that $v \notin W$ (by definition of $I$ ) and hence that $w \notin W$ (since $w={ }_{G} v$ ). So $\mathfrak{B}^{\prime}$ terminates if and only if its input $w$ does not lie in $W$ as required.

We now have our main result:

Theorem 12. If $G$ is a finitely generated group then the following are equivalent:

1. G has a recursively enumerable irreducible word problem with respect to every finite generating set.

2. G has a finite generating set with respect to which it has a recursively enumerable irreducible word problem and is loopy. 
3. G has a recursive word problem with respect to every finite generating set.

4. G has a recursive irreducible word problem with respect to every finite generating set.

Proof. $(1 \Rightarrow 2)$ Suppose $G$ has a recursively enumerable irreducible word problem with respect to every finite generating set. By Theorem 5 there is a finite generating set $A$ with respect to which $G$ is loopy. Since $G$ has a recursively enumerable irreducible word problem with respect to every finite generating set, it certainly has such an irreducible word problem with respect to $A$.

$(2 \Rightarrow 3)$ Suppose that $G$ is loopy with respect to a finite generating set $A$ and that $I_{A}(G)$ is recursively enumerable. By Theorem 6 we have that $P_{A}(g)$ holds in $\Gamma(G, A)$ for all $g$ in $G$. Since $I_{A}(G)$ is recursively enumerable Theorem 11 gives that $W_{A}(G)$ is recursive. As the word problem of $G$ being recursive is independent of choice of finite generating set, $G$ has a recursive word problem.

$(3 \Rightarrow 4)$ : This follows immediately from Proposition 8 .

$(4 \Rightarrow 1)$ : This follows immediately from the fact that a recursive language is recursively enumerable.

\section{Dependence on generating set}

In [5] a group was constructed whose word problem with respect to a particular finite generating set $A$ is recursively enumerable but not recursive. The basic construction uses small cancellation theory (see [13] for example) and proceeds as follows. Suppose that we have an alphabet $X=\left\{x_{1}, \ldots, x_{k}\right\}$ and suppose that $L \subseteq X^{*}$. Let

$$
A=\left\{x_{1}, \ldots, x_{k}, a_{1}, \ldots, a_{12}\right\},
$$

and define $R^{\prime}$ to be

$$
\left\{a_{1} \alpha \ldots a_{12} \alpha: \alpha \in L\right\} .
$$

We may then define $R$ to be the set of cyclically reduced words formed from $R^{\prime}$ by taking the closure under inverses and cyclic permutations and let $G$ be the group with presentation $\langle A: R\rangle$. Let $\Sigma=A \cup A^{-1}$. One can show that, for any word $u$ over $A$, we have that

$$
u \in L \Longleftrightarrow a_{1} u a_{2} u \ldots a_{12} u \in W_{A}(G)
$$


and that $I_{A}(G)$ is the closure of $R^{\prime}$ under taking inverses and cyclic permutations. If $L$ is a language that is recursively enumerable but not recursive then $I_{A}(G)$ and $W_{A}(G)$ are both recursively enumerable but not recursive. So we have:

Proposition 13. There is a group with a finite generating set with respect to which the irreducible word problem is recursively enumerable but not recursive.

If such a group $G$ had a recursively enumerable irreducible word problem with respect to every finite generating set then, by Theorem 12, $G$ would have a recursive word problem, a contradiction. So, if we combine this example from [5] with the results of the present paper, we can deduce the following:

Corollary 14. The recursive enumerability of irreducible word problems is not independent of the choice of finite generating set.

Remark 15. Consider a finitely generated group $G$ as in Corollary 14 where we have two finite generating sets $A$ and $B$ for $G$ such that $I_{A}(G)$ is recursively enumerable but $I_{B}(G)$ is not recursively enumerable. By Proposition 10 we have that $W_{A}(G)$ is recursively enumerable; given that the word problem of a group being recursively enumerable is independent of the choice of finite generating set, we have that $W_{B}(G)$ is also recursively enumerable. So the converse of Proposition 10 does not hold (given that $W_{B}(G)$ is recursively enumerable but $I_{B}(G)$ is not).

It would be interesting to determine more about the class of groups where the irreducible word problem is recursively enumerable with respect to some finite generating set but where this depends on the choice of generating set.

\section{References}

1. Anisimov, A.V.: Group languages. Kibernetika 4 (1971) 18-24

2. Boone, W.W.: The word problem. An. of Math. 70 (1959) 207-265

3. Boone, W.W., Higman, G.: An algebraic characterization of groups with a solvable word problem. J. Austral. Math. Soc 18 (1974) 41-53

4. Dunwoody, M.J.: The accessibility of finitely presented groups. Invent. Math. $\mathbf{8 1}$ (1985) 449-457

5. Fonseca, A.R.: Formal languages and the irreducible word problem in groups. PhD thesis, University of Leicester (2005)

6. Fonseca, A.R., Parkes, D.W., Thomas, R.M.: Irreducible word problems in groups. In Campbell, C.M., Quick, M.R., Robertson, E.F., Smith, G.C., eds.: Groups St Andrews 2005 Volume 1. LMS Lecture Notes Series 339, CUP (2007) 327-340 
7. Fonseca, A.R., Thomas, R.M.: Context-free irreducible word problems in groups. In Fine, B., Gaglione, A.M., Spellman, D., eds.: Combinatorial Group Theory, Discrete Groups, and Number Theory. Contemporary Mathematics 421, American Mathematical Society (2006) 125-136

8. Haring-Smith, R.H.: Groups and simple languages. Trans. Amer. Math. Soc. 279 (1983) 337-356

9. Herbst, T., Thomas, R.M.: Group presentations, formal languages and characterizations of one-counter groups. Theoret. Comp. Sci. 112 (1993) 187-213

10. Higman, G.: Subgroups of finitely presented groups. R. Soc. Lond. Philos. Trans. Ser. A Math. Phys. Eng. Sci. 262 (1961) 455-475

11. Ito, M., Kari, L., Thierrin, G.: Insertion and deletion closure of languages. Theoret. Comp. Sci. 183 (1997) 3-19

12. Lakin, S.R., Thomas, R.M.: Context-sensitive decision problems in groups. In Calude, C.S., Calude, E., Dinneen, M.J., eds.: Developments in Language Theory: $8^{\text {th }}$ International Conference. LNCS 3340, Springer-Verlag (2004) 296-307

13. Lyndon, R.C., Schupp, P.E.: Combinatorial group theory. Ergebnisse der Mathematik und ihrer Grenzgebiete. Springer-Verlag (1977)

14. Madlener, K., Otto, F.: About the descriptive power of certain classes of finite string-rewriting systems. Theoret. Comp. Sci. 67 (1989) 143-172

15. Muller, D., Schupp, P.: Groups, the theory of ends, and context-free languages. J. Comput. System Sci. 26 (1983) 295-310

16. Novikov, P.S.: On the algorithmic unsolvability of the word problem in group theory. Trudy. Mat. Inst. Steklov 44 (1955) 1-143

17. Parkes, D.W., Shavrukov, V.Y., Thomas, R.M.: Monoid presentations of groups by finite special string-rewriting systems. Theor. Inform. Appl. 38 (2004) 245-256

18. Parkes, D.W., Thomas, R.M.: Groups with context-free reduced word problem. Comm. Algebra 30 (2002) 3143-3156 